\title{
Tanto la varenidina como el bupropion utilizados para dejar de fumar no mostraron mayor riesgo de depresión, conducta auto-agresiva o suicidio comparado con el uso de terapia de reemplazo nicotínica
}

Both varenicline and bupropion used for smoking cessation showed no increased risk of depression, self-harm or suicide behavior compared with the use of nicotine replacement therapy

Thomas KH y col. BMJ 2013;347:1-9

\section{Objetivo}

Comparar el riesgo de suicidio, conductas auto-agresivas y depresión en pacientes medicados con vareniclina o bupropion contra aquellos que recibieron terapia de reemplazo nicotínica (TRN).

\section{Diseño, lugar y pacientes}

Estudio de cohorte prospectivo realizado en 349 centros de atención primaria del Reino Unido entre 2006 y 2011. Se incluyeron 119.546 pacientes, de los cuales 81.545 recibieron TRN, 6.741 bupropion y 31.260 vareniclina. El período de seguimiento de los pacientes fue de tres meses.

\section{Medición de resultados principales}

Se registraron los episodios depresivos, tomando como referencia la fecha en la cual el paciente inició tratamiento con un fármaco antidepresivo, y las conductas auto-agresivas a través del registro de admisiones hospitalarias por este problema y registros de mortalidad asociados a suicidios.

\section{Resultados principales}

Los principales resultados se detallan en la tabla 1. El análisis de sensibilidad a los seis y nueve meses mostró resultados similares.

Tabla 1. Riesgo de suicidio, conducta auto-agresiva y depresión en pacientes medicados con vareniclina, bupropion y terapia de reemplazo nicotínica a los tres meses del inicio de tratamiento.

\begin{tabular}{|c|c|c|c|c|}
\hline Medicación para dejar de fumar & $\begin{array}{l}\text { Total de } \\
\text { personas-año }\end{array}$ & $\begin{array}{l}\text { Nro. de eventos/ } \\
\text { Nro. de pacientes medicados }\end{array}$ & $\begin{array}{l}\text { HR (IC 95\%) } \\
\text { Ajuste básico* }\end{array}$ & $\begin{array}{c}\text { HR (95\%CI) } \\
\text { Ajuste completo* * }\end{array}$ \\
\hline \multicolumn{5}{|l|}{ Suicidio y conducta auto-agresiva } \\
\hline Reemplazo nicotínico & 19.196 & $69 / 78.407$ & 1 & 1 \\
\hline Bupropion & 1.622 & $4 / 6.568$ & $0,62(0,22 \mathrm{a} 1,70)$ & $0,83(0,30$ a 2,31) \\
\hline Vareniclina & 7.363 & $19 / 30.352$ & $0,70(0,41$ a 1,18$)$ & $0,88(0,52$ a 1,49$)$ \\
\hline \multicolumn{5}{|l|}{ Depresión en tratamiento } \\
\hline Reemplazo nicotínico & 10.315 & $799 / 42.475$ & 1 & 1 \\
\hline Bupropion & 961 & $40 / 3.910$ & $0,56(0,41$ a 0,77$)$ & $0,63(0,46$ a 0,87$)$ \\
\hline Vareniclina & 4.435 & 255/18.386 & $0,69(0,60$ a 0,80$)$ & $0,75(0,65$ a 0,87$)$ \\
\hline
\end{tabular}

HR: hazard ratio. IC 95\%: intervalo de confianza del 95\%. * Ajustado por edad, sexo y año de primera prescripción. ** Ajustado por sexo, edad, enfermedad psiquiátrica previa, uso previo de psicofármacos, antecedente de conducta autoagresiva, nivel socioeconómico, uso previo de medicamentos para dejar de fumar, año de primera prescripción y consultas en el año previo a la prescripción.

\section{Conclusiones}

El estudio no mostró evidencia de un mayor riesgo de depresión, conductas auto-agresiva o suicidio en los pacientes medicados con vareniclina o bupropion comparado con aquellos que recibieron TRN. Los resultados hallados son útiles para aumentar la confianza en la utilización de medicamentos para dejar de fumar tanto en los pacientes como en los prescriptores.

Fuente de financiamiento: Medicines and Healthcare Products Regulatory Agency (agencia gubernamental del Reino Unido). Conflictos de interés: los autores no reportan otros conflictos de intereses más allá de percibir honorarios de la previamente mencionada agencia.

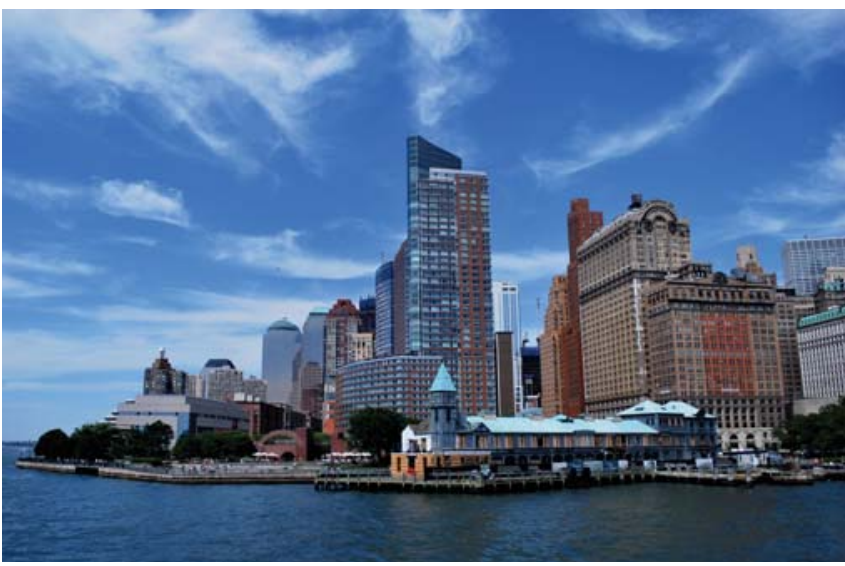




\section{Comentario}

El consumo de tabaco mata a más de cinco millones de personas al año y es responsable de la muerte de uno de cada diez adultos, constituyendo la primera causa de muerte prematura evitable en el mundo'. El uso de tratamientos farmacológicos como parte de la intervención profesional en los pacientes fumadores duplica o triplica las posibilidades de dejar de fumar. Los fármacos de primera línea para esta indicación son la TRN, el bupropion y la vareniclina, este último aprobado en el 2006 por la Agencia de Drogas y Alimentos de los EE. UU. (sigla en inglés, FDA) ${ }^{2}$. Con respecto a la vareniclina, los reportes realizados por la FDA en relación a la posibilidad de aparición de alteraciones neuropsiquiátricas, tales como cambios de conducta, hostilidad, agitación, estado anímico depresivo e ideas o actos suicidas, han generado cierta incertidumbre entre los profesionales en relación a su seguri$\mathrm{dad}^{3}$. Por su parte, el bupropion y otros antidepresivos tienen advertencias de la FDA sobre el aumento en el riesgo de experimentar pensamientos y comportamientos suicidas durante el tratamiento inicial en niños y adultos jóvenes ${ }^{4}$.

El presente estudio contribuye con información sobre la relación de estos fármacos en la aparición de depresión, conductas auto-agresivas y suicidio. Los resultados coinciden con los publicados por otros autores que no hallaron asociación con las variables mencionadas tanto para vareniclina como bupropion$^{5}$. Una de las críticas al presente trabajo es la definición del diagnóstico de depresión utilizando como proxy al inicio de tratamiento farmacológico antidepresivo. Cabe destacar que la mayoría de los antidepresivos más utilizados no solo se indican para el tratamiento de los cuadros depresivos sino también para otras patologías como los trastornos de ansiedad. Vale aclarar que en el Reino Unido el bupropion no está aprobado para el tratamiento de la depresión, limitándose su uso a la cesación tabáquica.

\section{Conclusiones del comentador}

Las medicaciones para dejar de fumar ayudan principalmente en la disminución de los síntomas de abstinencia, aumentando de esta manera las posibilidades de éxito en el intento de cesación. Los profesionales de la salud debemos indicarlas en todos los fumadores (salvo algunas excepciones) que quieran dejar de fumar considerando los sustanciales beneficios para la salud. La indicación de bupropion y vareniclina, a diferencia de la TRN, requieren un monitoreo estrecho por el profesional que los indica, y en aquellos pacientes con antecedentes psiquiátricos este control deberá ser más intensivo. Al ser una medicación más nueva que el bupropion, aún hacen falta más estudios para conocer los efectos en el comportamiento de la vareniclina.

Cecilia Jorgelina De Simone [ Servicio de Psiquiatría y Programa de Control del Tabaco del Hospital Italiano de Buenos Aires cecilia.desimone@ hosptitalitaliano.org.ar ]

De Simone CJ. Tanto la vareniclina como el bupropion utilizados para dejar de fumar no mostraron mayor riesgo de depresión, conducta auto-agresiva o suicidio comparado con el uso de terapia de reemplazo nicotínica. Evid Act Pract Ambul. 2016;19(2):53-54. Abr-Jun. Comentado de: Thomas $\mathrm{KH}$, y col. Smoking cessation treatment and risk of depression, suicide, and self harm in the Clinical Practice Research Datalink: prospective cohort study. BMJ. 2013;347(f5704). PMID: 24124105

\section{Referencias}

1. Organización mundial de la salud (página Web en internet). ¿Por qué el tabaco es una prioridad de salud pública?. (actualizada 2015; consulta 9/09/2015). Disponible en: http://www.who.intttobacco/health_priority/es/

2. Saimovici J. Farmacoterapia para dejar de fumar. En: GRANTAHI. Programa control del tabaco Hospital Italiano. Editora: Bakalar, Karina. Tratamiento del Tabaquismo. 1ra edición. Buenos Aires: delhospitalediciones; 2013:195-214

3. U.S. Food and Drug Administration (página Web en internet). ) U.S. Department of Health and Human Services. Comunicado de la FDA sobre la seguridad de los medicamentos: Información actualizada sobre la revisión de seguridad de Chantix (vareniclina) y el riesgo de efectos neuropsiquiátricos adversos. (actualizada 24/10/2011; consulta 13/09/2015). Disponible en: http://www.fda.gov/Drugs/DrugSafety/ucm278497.htm

4. U.S. Food and Drug Administration (página Web en Internet) U.S. Department of Health and Human Services. FDA Requires New Boxed Wamings for the Smoking Cessation Drugs Chantix and Zyban. (actualizada 1/07/2009; consulta 11/09/2015). Disponible en: http://www.fda.gov/Drugs/DrugSafety/DrugSafetyPodcasts/ucm170906.htm

5. Molero Y, y col. Varenicline and risk of psychiatric conditions, suicidal behaviour, criminal offending and transport accidents and offences: population based cohort study. BMJ $2015 ; 350: 1-7$.

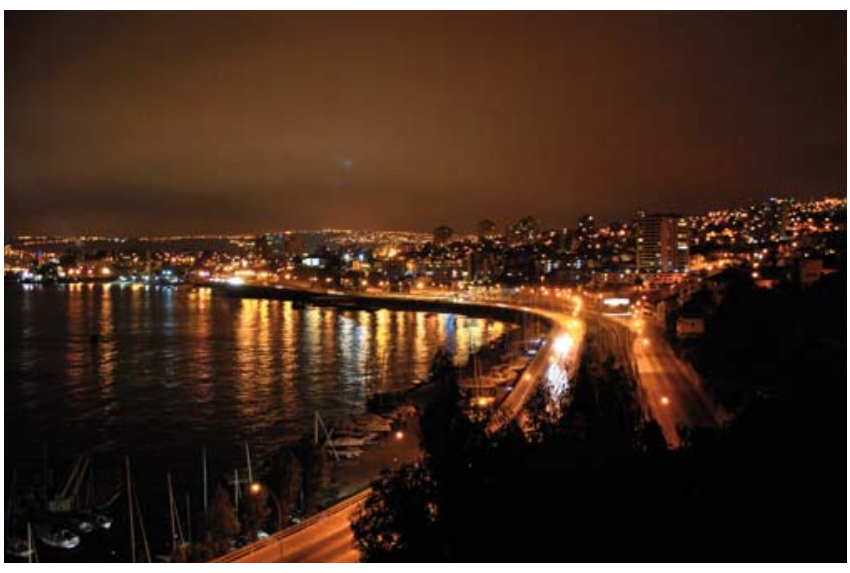

